\section{MHC molecular mimicry in diabetes}

To the editor - The recent News and Views article by Solimena and De Camilli ${ }^{1}$, and the associated Letter to the Editor by Jones and Armstrong $^{2}$, consider molecular mimicry (see also Albani et al., p. XXX, this issue) as a proposed mechanism underlying insulindependent diabetes mellitus (IDDM). In particular, they focus on the amino-acid sequence, PEVKEK, found in both glutamic acid decarboxylase (GAD65, a key islet autoantigen in IDDM $^{3}$ ) and in the Coxsackie B virus (an environmental agent putatively involved in the pathogenesis of IDDM).

We would like to draw attention to a similarity between a different peptide of the Coxsackie $B$ virus and another islet autoantigen, carboxypeptidase $\mathrm{H}$, autoantibodies to which have also been claimed to be characteristic of IDDM ${ }^{4}$. However, we wish to point out this similarity not so much in the context of arbitrary pathogen-autoantigen mimicry, but in relation to two questions not yet addressed in this discussion.

The first of these is which 'sequence similarity peptides' (ssp) are relevant to autoimmune processes? (As pointed out by Jones and Armstrong', GAD65 has many regions of sequence similarity to virus proteins, but subjects with IDDM did not exhibit significant $\mathrm{T}$-cell responses to most of the corresponding viruses.) The second, and probably related, question is why many autoimmune diseases, including IDDM, are restricted to subjects with particular HLA haplotypes? We have previously suggested ${ }^{5,6}$ that an underlying mechanism for the initiation of autoimmune disease may be three-way molecular mimicry between MHC-derived selfpeptides (of class I or class II origin) presented by $\mathrm{MHC}$ class II antigens, and ssps of infectious agents and/or of characteristic autoantigens. In studies on peptide

Coxsackievirus b4, coat protein VP1156:

HLA-DQ 3.2, $\beta$ chain

Carboxypeptidase $\mathrm{H}$, human
3:

230: presentation by class II molecules, MHC peptides of both classes are eluted with high frequency". Under this "MHCmay initiate an autoimmune response. $T$ cells stimulated by foreign peptides may recognize sequence similarities in $\mathrm{MHC}$ derived self-peptides presented on target tissues by "inappropriately" expressed class II antigens (for example, following a mild viral infection). Alternatively, $\mathrm{T}$ cells with moderate affinity for self-MHC peptides are selected when these are presented during thymic development, and allowed into the periphery. These cells become primed for an autoimmune response when autoantigenic and/or foreign peptides with sequence similarities are presented with appropriate co-signals. In this hypothesis, MHC linkage with disease resides in both the primary structure of class I and class II alleles that give rise to the ssps, and in any class II restriction on their presentation.

In the case of IDDM, we have already pointed out ${ }^{7}$ that there is a remarkable similarity between a peptide of the unique class II molecule (I-A $\mathrm{A}^{8^{7}}$ ) of the NOD mouse, and the peptide commencing at amino acid 509 of GAD65, already referred to by Jones and Armstrong as the dominant T-cell epitope for IDDM in this animal model. (It is highly pertinent that $100 \%$ diabetes incidence only occurs in NOD colonies reared under specific pathogen-free conditions, which suggests that diabetes development is an entirely endogenous process). Modelling studies, based on $\mathrm{I}-\mathrm{A}^{\mathrm{g} 7}$ binding motifs, have indicated that these I- $\mathrm{A}^{\mathrm{g} 7}$ and GAD65 ssps would bind tightly to this class II molecule. Moreover, preliminary experiments that we have performed have indicated that the onset of diabetes in female NOD mice may be delayed or prevented by immunization at 3 weeks of age with the I-A $A^{g^{7}} \operatorname{ssp}^{8}$. Further preliminary experiments indicate that in four out of eight of the long-term surviving mice, protection from diabetes was overcome within days by administration of 1,000 units of recombinant interleukin-2, a manoeuvre known to break immunological tolerance (Gearon, Peakman, Davies, Vermimicry" hypothesis, one of two scenarios

gani \& Baum, unpublished observations).

With that background, it seems very significant that the ssps of Coxsackie B virus and carboxypeptidase $\mathrm{H}$ also strongly resemble a sequence in the $\beta$-chain of HLADQ 3.2 in the figure. That allele is known to confer susceptibility to IDDM $^{9}$. While the sequence is not unique to that particular DQ antigen it is of interest that this particular peptide, derived from HLA-DQ 3.2, was the only class II peptide reported in a particular study as being eluted from HLA-DR4, another susceptibility allele for IDDM ${ }^{9}$.

We do not wish to imply that the set of mimicries illustrated in the figure is unique in relation to the possible pathogenesis of IDDM. (For example, a peptide of the nucleoprotein of influenza $A$ virus has the ssp: 452-SPEDVSFQG.) What we do wish to stress in the consideration of the role of autoantigens, HLA molecules and putative infective agents in the aetiology of autoimmune diseases, is the possibility of the restricted presentation by MHC class II antigens of cross-reacting ssps of susceptibility alleles.

1. Solimena, M. \& De Camilli, P. Coxsackieviruses and diabetes. Nature Med. 1, 25-26 (1995).

2. Jones, D.B. \& Armstrong, N.W. Coxsackie virus and diabetes revisited. Nature Med. 1, 284 (1995).

3. Baekkeskov, S. et al. Identification of the $64 \mathrm{~K}$ au toantigen in insulin-dependent diabetes as the GABA synthesizing enzyme glutamic acid decarboxylase. Nature 347, 151-156, (1990).

4. Castaño, L., Russo, E., Zhou, L., Lipes, M.A. \& Eisenbarth, G.S. Identification and cloning of a granule autoantigen (carboxypeptidase $\mathrm{H}$ ) associated with type 1 diabetes. J. clin. Endocrinol. Metab. 73, 1197-1201 (1991).

5. Baum, H., Butler, P., Davies, H., Sternberg, M. \& Burroughs, A. Autoimmune disease and molecular mimicry: an hypothesis. Trends biochem. Sci. 18, 140-144 (1993).

6. Chicz, R.M. et al. Specificity and promiscuity among naturally processed peptides bound to HLA-DR alleles. J. exp. Med. 178, 27-47 (1993).

7. Baum, H. \& Cunningham, P. Do MHC antigens generate pathogenetic peptides? Immun. Today 15 388-389 (1994).

8. Gearon, C.L., Peakman, M., Vergani, D., \& Baum $\mathrm{H}$. Mimicry between an islet autoantigen and MHC class II molecules as a mechanism of autoimmune diabetes. Diabetic Med.(in the press).

9. Todd, J.A. Genetic control of autoimmunity in type 1 diabetes. Immun. Today 11, 122-128 (1990).

Harold Baum ${ }^{1}$, Vladimir Brusic ${ }^{2}$, Kaushik Choudhuri ${ }^{3}$, Phillp CunningHAM $^{1}$, DiEgo Vergani ${ }^{3}$, Mark PEAKMAN ${ }^{3}$

'Division of Life Sciences, King's College London, Campden Hill Road, London W8 7AH, UK,

'The Walter and Eliza Hall Institute of Medical Research, Royal Melbourne Hospital, Melbourne Victoria 3050, Australia ${ }^{3}$ Department of Immunology, King's College School of Medicine \& Dentistry, Bessemer

"Sequence similarities between an HLA-DQ3.2 peptide (binds to HLA-DR4) and the Coxsackie virus b4, coat protein VP1, and the carboxypeptidase $H$. Identical or conserved residues fall within the boxed region.
Road, London SE5 9PJ, UK 